\title{
CF-targeter: a rational biological cell factory targeting platform for biosynthetic target chemicals
}

Shaozhen Ding ${ }^{1}$, Pengli Cai ${ }^{1}$, Le Yuan², Yu Tian², Weizhong Tu ${ }^{3}$, Dachuan Zhang ${ }^{1}$, Xingxiang Cheng ${ }^{1}$, Dandan

Sun ${ }^{1}$, Junni Chen ${ }^{3}$ and Qian-Nan $\mathrm{Hu}^{1 *}$

${ }^{1}$ CAS Key Laboratory of Computational Biology, CAS-MPG Partner Institute for Computational Biology, Shanghai Institute of Nutrition and Health, Shanghai Institutes for Biological Sciences, University of Chinese Academy of Sciences, Shanghai, 200333, P. R. China

2 Tianjin Institute of Industrial Biotechnology, Chinese Academy of Sciences, Tianjin 300308, People's Republic of China.

${ }^{3}$ Wuhan LifeSynther Science and Technology Co. Limited, Wuhan, 430070, People's Republic of China

Details of several pathway-finding tools that were mentioned in the review (https://doi.org/10.1186/s13321-017-0239-6) are shown below. In conclusion, most of these methods do not use genome-scale metabolic models, so they could not be utilized to calculate carbon fluxes. Some of these methods utilized reaction rules that are not key points of our research. On the other hand, unlike some of these methods that could retrieve pathways between two specified molecules within metabolic networks of one or more chassis hosts, the algorithm that was utilized in this research allows for automatic search without knowing a precursor in the host. All these algorithms have advantages, and they can solve some of the problems in synthetic biology from different aspects. We have also fully proven that the pathway search algorithm that was used in this research could produce desired results in both the current study and in a previous study (DOI:

10.1021/acschembio.7b00605)

Table S1 : Chassis-related pathway-finding tools mentioned in the review

\begin{tabular}{|c|c|c|c|c|c|}
\hline Tools & $\begin{array}{c}\text { Only need } \\
\text { target }\end{array}$ & GEM-model used & $\begin{array}{c}\text { Flux } \\
\text { control }\end{array}$ & $\begin{array}{c}\text { Reactions } \\
\text { rules }\end{array}$ & Comments \\
\hline Masanori Arita[1] & no & no & no & no & {$[\mathrm{a}]$} \\
\hline Rahnuma[2] & no & no & no & no & {$[\mathrm{b}]$} \\
\hline MRSD[3] & no & no & no & no & {$[\mathrm{c}]$} \\
\hline Sunwon Park[4] & yes & no & no & yes & {$[\mathrm{d}]$} \\
\hline RouteSearch [5] & yes & no & no & no & {$[\mathrm{e}]$} \\
\hline DESHARKY [6] & yes & yes & yes & no & {$[\mathrm{f}]$} \\
\hline GEM-path[7] & yes & yes & yes & yes & {$[\mathrm{g}]$} \\
\hline MRE[8] & no & no & no & no & {$[\mathrm{h}]$} \\
\hline
\end{tabular}

[a]: By using an atomic tracer and weighting schema in which $E$. coli native reactions have priority, this tool could help to retrieve pathways between the two molecules in Escherichia coli intermediary metabolism, so it could be used for E. coli metabolism reconstruction. This tool does not utilize the $E$. coli genome-scale metabolic model but uses E. coli metabolism data from EcoCyc, so there is no flux control, as was explained; thus, the theoretical yield could not be calculated.

[b-c]: Both tools require the user to select which organism or group of organisms to observe. To be specific, these two tools could help to find pathways between two molecules by using reactions from one or more organisms; the microbial information and enzymatic reactions are from the KEGG database. Our initial purpose for selecting a pathway search algorithm is to identify heterologous reactions for the specified target molecule production 
when engineering one microbial host. On the other hand, both tools utilized reactions from KEGG, and they do not use genome-scale metabolic models, so the outputs could not be utilized to calculate carbon fluxes in the next step.

[d]: By using 50 reaction rules, this tool helps to predict a novel synthetic pathway for the production of desired chemicals. The results are filtered by several categories, including chemical similarity, thermodynamic favorability, pathway distance, organism specificity and so on. For part of the organism specificity, it estimates the organism-to-organism distance, which is calculated by the hierarchical information of organism lineages based on gene changes from generation to generation. The lineage information of all genes comes from the KEGG database. The approach that is utilized in organism specificity could be regarded as an approach for pathway-ranking; we do not call it a pathway-finding method for the reason that an enzyme route candidate has already been determined before this step.

[e]: Based on an organism native database (e.g., EcoCyc) and a database that is identified as a library of compounds and reactions (e.g., MetaCyc), this tool could help to find a biosynthetic pathway between two molecules by using atom mappings, and the native compounds and reactions are assumed to take precedence. Therefore, this tool could help to distinguish native parts from the heterologous path within the whole pathway. However, data on the native metabolism of the microbial host is from MetaCyc or EcoCyc, rather than a genome-scale model. Thus, there is no flux control, and the outputs could not be used for theoretical yield calculation.

[f]: By using an E. coli genome-scale metabolic model and flux balance analysis, the tool named DESHARKY could help to retrieve heterologous pathways for target molecule production when engineering E. coli, and then the results could be used to calculate carbon fluxes. Similar to the algorithm used in ref. 15, this method could also be expanded to other microbial chassis.

[g]: By using reaction rules, Palsson and his colleagues developed a tool named GEMPath, which could be utilized to retrieve biosynthetic pathways for 20 large-volume compounds production within 4 reactions from the metabolic network of $E$. coli. Because GEM-Path used reaction rules, the pathways may contain novel reactions, which is not a key point of our research. On the other hand, there may be many pathways when using reaction rules, so GEM-Path only explores pathways that have a length of no more than 4. However, the algorithm that was used in our article is able to explore pathways that have a length of no more than 10.

[h]: MRE is a web tool that could help to suggest foreign enzymes for a biosynthesis pathway design with competing endogenous reactions in mind. Data on microbial metabolism in MRE came from KEGG, rather than the genome-scale metabolic model. Therefore, there is no flux control, and the results could not be used to calculate the theoretical yield.

[1]: Masanori Arita. In Silico Atomic Tracing by Substrate-Product Relationships in Escherichia coli Intermediary Metabolism. Genome Research.

[2]: Aziz Mithani, Gail M. Preston and Jotun Hein1.Rahnuma: hypergraph-based tool for metabolic pathway prediction and network comparison. Bioinformatics.

[3]: Deguo Xia, Haoran Zheng. MRSD: a web server for Metabolic Route Search and Design. Bioinformatics.

[4]: Ayoun Cho, Hongseok Yun. Prediction of novel synthetic pathways for the production of desired chemicals. BMC Systems Biology.

[5]: Mario Latendresse, Markus Krummenacker, and Peter D. Karp. Optimal Metabolic Route Search Based on Atom Mappings. Bioinformatics

[6]: Guillermo Rodrigo, Javier Carrera. DESHARKY: automatic design of metabolic pathways for optimal cell growth. Bioinformatics

[7]: Bernhard O. PalssonAdam M. Feist. Generation of an atlas for commodity chemical production in Escherichia coli and a novel pathway prediction algorithm, GEM-Path. Metabolic Engineering.

[8]: Hiroyuki Kuwahara† MRE: a web tool to suggest foreign enzymes for the biosynthesis pathway design with competing endogenous reactions in mind. Nucleic Acids Research. 


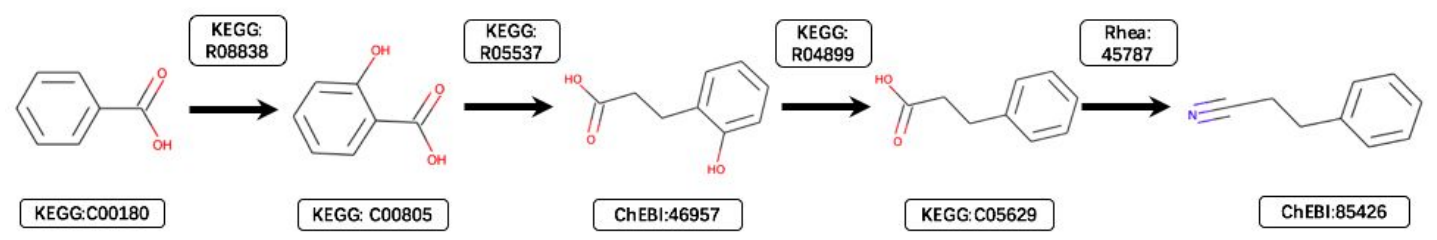

Figure S1 : Biosynthetic pathway found in this research

According to our statistics, there are 1,514 reactions that existing only in Rhea, but not in KEGG. Therefore, pathways that contain these reactions could not be found on the KEGG pathway map. Herein, we give an example (Figure S1). Figure S1 shows the biosynthetic pathway for producing phenylpropanonitrile from benzoate. The pathway consists of four reactions, in which the first three reactions only exist in KEGG, and the last reaction only exists in Rhea. The whole pathway could neither be found in the KEGG pathway map, nor in Rhea. However, it could be found when merging the two databases. 